\title{
Prevalencia de sensibilización a látex en personal de pabellones quirúrgicos del Hospital Clínico de la Universidad de Chile
}

\author{
M Antonieta G uzmán $\mathrm{M}^{1}$, Virginia Arancibia $\mathrm{E}^{2}$, \\ Jessica Salinas L $^{1}$, Claudia Rodas $E^{1}$, Johanna Roa $A^{1}$, \\ Rodrigo Villegas $\mathbf{R}^{3 a}$. \\ Prevalence of latex hypersensitivity \\ in operating room workers of the \\ University of Chile Clinical Hospital
}

Background: Health care workers (HCW) are a high risk group for developing natural rubber latex (NRL) hypersensitivity and allergy. Some studies showed a correlation between time and frequency of exposure to NRL gloves and hypersensitivity, but a recent meta-analysis showed no clear evidences for such assumption. Aim: To determine the prevalence of NRL hypersensitivity and allergy in a group of HCW at the University of Chile Clinical Hospital. Materials and Methods: Ninety five HCW (aged $37 \pm 10$ years, 59 females) were interviewed about time of exposure, atopic diseases and latex-related allergy symptoms. Different NRL extracts and seven NRL gloves brands were tested by the prick test method. Results: Twenty four workers (25\%, $95 \% \mathrm{CI}=16.9 \%-35.2 \%)$ were sensitized. No gender differences were found. No symptomatic cases were found in the sensitized group. In the workplace, six and two non sensitized subjects had respiratory symptoms or contact urticaria, respectively. Sensitivity to bananas, avocadoes, kiwi and chestnut was not significantly more common among latex sensitive individuals. No differences between sensitized and non sensitized subjects were observed for the presence of atopic diseases or the mean number of years at the workplace. The sensitization rate to NRL increased along with years of work, from $18.6 \%$ in patients working less than 10 years, to $28.1 \%$ in the group between 10 and 20 years and to $35 \%$ in those working more than 21 years ( $p=0.693$ ). Sensitization was also related to the weekly hours of exposure: 1-10 hours, $17 \%$; $11-20$ hours, $23.5 \%$; $21-30$ hours, $50 \%$ and $31-40$ hours, $28.5 \%(\mathrm{p}=0.036)$. Conclusions: Sensitization increased proportionally to the years and weekly hours wearing NLR gloves. We did not find symptomatic patients in the sensitized group (Rev Méd Chile 2005; 133: 535-40).

(Key Words: Environmental illness; Latex hypersensitivity; Multiple chemical sensitivity)

\footnotetext{
Recibido el 9 de junio, 2004. Aceptado en versión corregida el 14 de marzo, 2005.

${ }^{1}$ Centro de Alergias, Sección Inmunología, Hospital Clínico de la Universidad de Chile. ${ }^{2}$ Departamento de Anestesiología y Reanimación, Hospital Clínico de la Universidad de Chile. ${ }^{3}$ Escuela de Salud Pública, Facultad de Medicina, Universidad de Chile. Santiago de Chile. ${ }^{a}$ Estadístico.
}

Correspondencia a: M. Antonieta Guzmán M. Centro de Alergias, Sección Inmunología, Hospital Clínico Universidad de Chile. Santos Dumont $999,5^{\circ}$ piso, sector E, Independencia. Santiago. Fax: 56-2-6788567E. E mail: mguzman@redclinicauchile.cl. 
$\mathrm{L}$ a alergia al látex constituye hoy en día un problema médico importante, que afecta cada vez a un mayor número de pacientes. La primera descripción de hipersensibilidad inmediata por látex se realizó en Alemania ${ }^{1}$, apareciendo la siguiente cincuenta y dos años después ${ }^{2}$. Desde esa fecha, son cada vez más numerosos los casos publicados de reacciones alérgicas a productos de caucho natural, incluyendo anafilaxias sistémicas.

El látex o caucho natural es un producto vegetal procesado, que se obtiene del jugo lechoso de una variedad de árboles cauchíferos, en especial Hevea brasiliensis, Ficus elástica y Parthenium argentatum. En la composición del látex participan polímeros de la goma, proteínas, carbohidratos, lípidos y otros compuestos ${ }^{3}$. El látex es ubicuo y está presente en productos de uso cotidiano y profesional. Estos productos ven aumentada su potencial alergenicidad, debido a los diversos procesos de fabricación comercial a los que es sometido el látex ${ }^{4}$.

Existen grupos de riesgo de sensibilización al látex, la que ocurre principalmente por mecanismos de hipersensibilidad inmediata, mediados por IgE, contra alergenos pertenecientes a la fracción proteica del látex natural y por hipersensibilidad retardada, en cuyo caso la respuesta es de tipo linfocitaria y se dirige contra los aditivos agregados al látex durante su proceso de fabricación ${ }^{5}$, con el posterior desarrollo de síntomas clínicos. La sintomatología, en el caso de la hipersensibilidad inmediata, se traduce en cuadros de urticaria de contacto, angioedema, rinoconjuntivitis, asma y reacciones anafilácticas ante la exposición a elementos de látex ${ }^{5}$. Se ha reportado también la existencia de una bronquitis eosinofílica en una enfermera ${ }^{6}$. El síntoma con mayor valor predictivo para el diagnóstico de alergia a látex es la urticaria de contacto en relación al uso de guantes y que se presenta en el lapso de minutos después de la exposición ${ }^{7,8}$. Se ha estimado que existe un promedio de 2 años para desarrollar asma por látex, una vez presentada la urticaria de contacto. Respecto a los síntomas de hipersensibilidad retardada, éstos se traducen en una dermatitis de contacto con el uso de elementos de látex, como los guantes $5,9,10$. Otro cuadro clínico muy frecuente es la dermatitis irritativa, que no se debe a un mecanismo de hipersensibilidad, pero que facilita el desarrollo de una alergia, al perderse la integridad cutánea y facilitarse la exposición a alergenos.
Los grupos de riesgo están representados por el personal de salud, los trabajadores de la industria de la goma, los niños con espina bífida y malformaciones urinarias, los pacientes multioperados, los individuos atópicos y los pacientes que presentan reacciones de hipersensibilidad inmediata con la ingesta o contacto con frutas de reactividad cruzada con el látex como palta, plátano, castaña y kiwi, entre otros ${ }^{11-13}$. Cifras internacionales describen $1 \%$ de sensibilización al látex en la población general, de 2 a $4 \%$ en atópicos y de hasta $15 \%$ en personal de salud ${ }^{14-18}$. En los pacientes con espina bífida, la sensibilización alcanza a $60 \%$ en algunas series. Además, se describen reacciones alérgicas graves en una de cada 5.000 cirugías $^{14}$, y el látex es el responsable en $15 \%$ de los casos, después de los relajantes musculares.

Aunque la exposición al látex se puede producir en cualquier lugar, las reacciones más graves se han descrito en los hospitales, tanto en personal sanitario como en sus pacientes ${ }^{19}$. De hecho, en la década 1980-89, despertó gran interés el aumento alarmante de reacciones anafilácticas intraoperatorias o durante exploraciones radiológicas, algunas de ellas con resultado mortal, que se atribuyeron a la exposición al látex ${ }^{20}$. En Estados Unidos de Norteamérica (EE.UU), los primeros casos se describieron a fines de esa década ${ }^{21}$ y en los cuatro años siguientes se comunicaron más de 1.000 reacciones adversas por látex, incluyendo 15 fallecimientos en relación con catéteres para enemas baritados. Por ello, la Food and Drug Administration (FDA) de EEUU, editó en 1990 una serie de recomendaciones acerca de la alergia al látex y retiró del mercado los catéteres para enemas con globos inflables de este material ${ }^{22}$.

Este incremento de la prevalencia de alergia al látex se ha explicado por el mayor uso de guantes de látex por parte del personal sanitario, como medida de protección contra los virus de inmunodeficiencia humana y hepatitis 23 . Otros factores a considerar son los cambios en el proceso de fabricación y de las cepas de Hevea (árbol cauchífero productor de látex) utilizadas. Por otra parte, la sustitución del talco por almidón de maíz, como lubricante de los guantes, proporciona un vehículo muy eficaz para los alergenos del látex, los que son liberados al ambiente con mayor facilidad, ya que la unión de los alergenos del látex con las partículas de almidón de maíz es más débil ${ }^{24,25}$. 
En Chile, un estudio de prevalencia realizado en personal de salud en 1997, en el Instituto Nacional del Tórax ${ }^{26}$, muestra $14,7 \%$ de sensibilización a látex.

La identificación de pacientes en riesgo es muy importante antes de plantear procedimientos que implican una exposición a látex, en este sentido, la historia clínica es fundamental ${ }^{27} \mathrm{y}$ debe preguntarse por síntomas sugerentes de reacciones previas, pertenencia a grupos de riesgo y eventuales estudios practicados y sus resultados. La historia clínica sugerente de hipersensibilidad inmediata debe ser complementada con pruebas cutáneas del tipo prick test. Pese a que los alergenos de Hevea brasiliensis no están completamente caracterizados, se considera que los prick test con látex o con extractos de sus productos son muy específicos, ya que las personas que no son alérgicas al látex presentan generalmente pruebas cutáneas negativas. Además, se considera que estas pruebas son muy sensibles, ya que casi la totalidad de las personas que han presentado una reacción alérgica al látex, presentan un prick test positivo con alguno de los extractos ${ }^{28}$, lo que es esperable si consideramos que antes de establecerse una reacción clínica mediada inmunológicamente, debe existir un período de sensibilización del individuo con los antígenos (alergenos) causales. Actualmente se considera a esta prueba como un método confiable, relativamente seguro (siempre debe practicarse en centros especializados en el tema) y que ofrece una sensibilidad y especificidad cercana al 100\% para efectuar el diagnóstico de sensibilización a látex ${ }^{29}$. La utilización simultánea de dos extractos distintos convenientemente validados para prick test, aproximaría la sensibilidad a $100 \% 30$.

La sospecha de dermatitis de contacto por látex debe estudiarse con test de parche que incluya los adititivos utilizados en el proceso de fabricación del guante, ya que rara vez la hipersensibilidad retardada se debe a alergenos propios del látex sin procesar $5,10,14$.

Los diversos métodos de diagnóstico in vitro (RAST, UniCAP, ELISA en placa, y otros) detectan las IgE específicas hasta en $70 \%$ de los casos, vale decir, su rendimiento diagnóstico es muy inferior al de las pruebas cutáneas ${ }^{30-32}$. De todos modos, son una herramienta diagnóstica de valor, especialmente en aquellas personas que presentan reacciones anafilácticas intraoperatorias recientes, en quienes inicialmente se practicará un estudio de IgE específica a látex, entre otros agentes causales, y si este resultado es negativo, podría plantearse una prueba cutánea para este alergeno, ya que ambas pruebas pueden ser complementarias. Las pruebas de provocación con látex son en teoría las pruebas diagnósticas definitivas pero no están exentas de riesgo y no existe un método de provocación con látex estandarizado internacionalmente. Además, pueden presentar falsos negativos y falsos positivos. Se acepta que están indicadas en caso de discordancia entre la historia clínica y los métodos complementarios (prueba cutánea en prick e IgE específicas en sangre). Estas pruebas también se pueden plantear para determinar el grado de sensibilización, como también con fines médico legales o de investigación.

Diversos estudios han planteado que el tiempo de exposición a látex correlaciona con el grado de sensibilización, pero un reciente metaanálisis concluye que la evidencia al respecto no es clara ${ }^{33}$.

El objetivo de este estudio fue determinar la prevalencia de sensibilización y de síntomas de alergia al látex en un grupo de personas de pabellones quirúrgicos del Hospital Clínico de la Universidad de Chile, y su relación con años y frecuencia de exposición al látex.

\section{Materiales y MÉTOdos}

En este estudio fueron enrolados como voluntarios 95 trabajadores de pabellones quirúrgicos del Hospital Clínico de la Universidad de Chile, que dieron su consentimiento informado (23 anestesistas, 11 cirujanos, 28 enfermeras, 33 auxiliares). Se realizó una extensa encuesta objetivando edad, sexo, tiempo de exposición, síntomas relacionados al uso de látex, antecedentes de enfermedades atópicas y síntomas en relación con frutas de la familia látex. Las respuestas al cuestionario fueron evaluadas por el investigador con cada voluntario. Se efectuaron prick test en la cara anterior del antebrazo con extracto comercial de látex (Leti, España), extracto de guantes de látex en solución salina (extracto preparado con 7 marcas de guantes de látex en solución salina, agitado en vórtex por $12 \mathrm{~h}$, concentración 1 gr en $20 \mathrm{ml}$ ), látex amoniacado (Madegom, Chile) y extractos comerciales de frutas relacionadas al látex: kiwi, castaña, palta, plátano (Leti, España). La lectura se realizó a los 15 min, considerando como un resultado positivo la presencia de una pápula cuyo diámetro promedio fuese mayor a $3 \mathrm{~mm}$ del control negativo (solución 
fisiológica); se utilizó como control positivo histamina $10 \mathrm{mg} / \mathrm{ml}$. Además se testearon siete diferentes marcas comerciales de látex con el método prick-prick (Guthrie, Prim-med, Sempermed, Top glove, Health pack, Medi gloves, Medi gloves sensi skin). Este estudio fue aprobado por el comité de ética de nuestro Hospital.

Estadística. Para variables cuantitativas se calculó el promedio y desviación estándar. Para variables cualitativas se calcularon porcentajes. A través del test de Chi Cuadrado se evaluó la asociación entre prevalencia y las otras características en estudio como género, enfermedades atópicas, años laborales y horas semanales de exposición. Para determinar diferencias de medias se usó el test t de Student. Se consideró un nivel de significación de 5\%.

\section{Resultados}

El total de participantes en este estudio fue de 95 trabajadores de pabellones quirúrgicos. Las características demográficas de los voluntarios y algunos datos de la encuesta se muestran en la Tabla 1.

El porcentaje de sensibilización total fue de $25,3 \%(n=24)(95 \%$ IC $=16,9 \%-35,2 \%)$, sin diferencias significativas según género $(\mathrm{F}=25,4 \%$ y $\mathrm{M}=25 \%)$ ( $p=0,963)$. El grupo de pacientes sensibilizados no mostró diferencias en relación con enfermedades atópicas en comparación con el grupo no sensibilizado $(54,2 \%$ y $53 \%$ respectivamente, $\mathrm{p}=0,956)$ ni en el promedio de años en las ocupaciones de cada grupo (13,5 $\pm 9,5$ años \pm DS y 19,3 $\pm 14,4$ años \pm DS, respectivamente). Por otro lado, tampoco se evidenció que la sensibilización a frutas de la familia látex (plátano, palta, kiwi y castaña) fuera más prevalente en los individuos sensibilizados al látex ( $\mathrm{p}=0,453)$.

Destaca que no se evidenciaron individuos sintomáticos dentro del lugar de trabajo en el grupo sensibilizado a látex. En el grupo de personas no sensibilizadas a látex, en cambio, 6 individuos $(6,3 \%)$ presentaron síntomas respiratorios (episodios de tos) en su lugar de trabajo y 2 individuos $(2,1 \%)$ presentaron episodios aislados de urticaria de contacto en relación a elementos de aseo (soluciones antisépticas). Se encontró un incremento en el porcentaje de sensibilización, desde 18,6\% en pacientes con menos de 10 años de exposición laboral, a 28,1\% en el grupo entre los 10-20 años laborales y a $35 \%$ en aquellos trabajadores con más de 21 años de ocupación ( $\mathrm{p}=0,693)$.

Se observó que el porcentaje de sensibilización se relacionó significativamente con las horas semanales de exposición al látex: 1-10 h con 17\%, $11-20$ h con $23,5 \%, 21-40$ h con $50 \%$ y $31-40$ h con $28,5 \%(\mathrm{p}=0,036)$.

\section{DisCUSIÓN}

La sensibilización al látex se ha convertido en un problema de creciente importancia en el personal de salud.

En nuestro estudio destaca el alto porcentaje de sensibilización al látex obtenido en el personal estudiado (25,3\%), superior a las cifras descritas en publicaciones extranjeras y nacionales.

Este alto porcentaje posiblemente se explique porque el estudio se practicó en los individuos expuestos a mayor concentración de látex ambiental, como es el grupo de trabajadores de la salud de pabellones quirúrgicos y no en personal hospitala-

Tabla 1. C aracterísticas del grupo estudiado

\begin{tabular}{|c|c|c|}
\hline & № & $\%$ \\
\hline Número de participantes & 95 & \\
\hline Edad: rango & $21-63$ & \\
\hline Promedio \pm DS & $37,1 \pm 9,7$ & \\
\hline \multicolumn{3}{|l|}{ Sexo } \\
\hline $\mathrm{F}$ & 59 & 62,1 \\
\hline M & 36 & 39,7 \\
\hline \multicolumn{3}{|l|}{ Enfermedades atópicas } \\
\hline Grupo sensibilizado & & 54,2 \\
\hline Grupo no sensibilizado & & 53,5 \\
\hline \multicolumn{3}{|l|}{ Duración de exposición } \\
\hline $0-10$ años & & 18,6 \\
\hline 11-20 años & & 28,1 \\
\hline 21-30 años & & 38,5 \\
\hline 31-40 años & & 28,6 \\
\hline \multirow{2}{*}{\multicolumn{3}{|c|}{$\begin{array}{l}\text { Contacto con guantes de látex: } \\
\text { h/sem }\end{array}$}} \\
\hline & & \\
\hline $0-10 \mathrm{~h}$ & & 17 \\
\hline $11-20 \mathrm{~h}$ & & 23,5 \\
\hline $21-30 \mathrm{~h}$ & & 50 \\
\hline $31-40 \mathrm{~h}$ & & 28,5 \\
\hline Sensibilización total & 24 & 25,3 \\
\hline $\mathrm{F}$ & 15 & 25,4 \\
\hline M & 9 & 25 \\
\hline
\end{tabular}




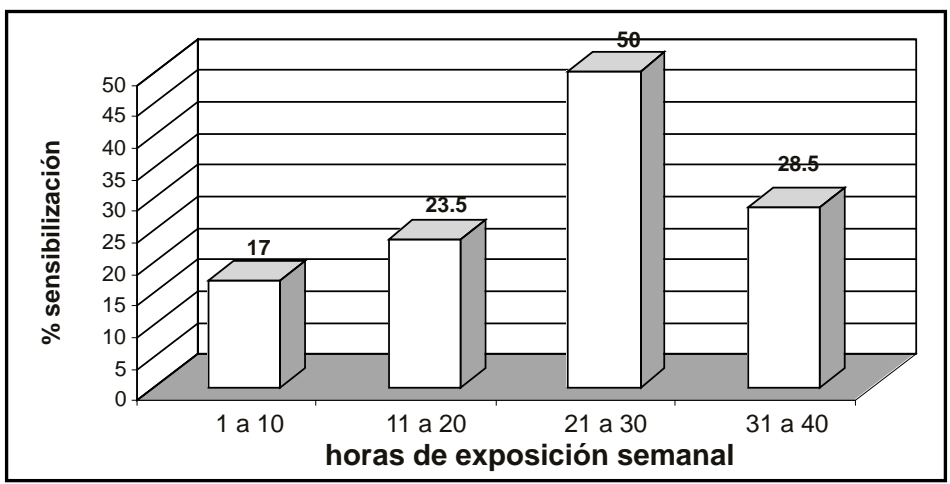

Figura 1. Porcentaje de trabajadores sensibilizados versus horas semanales de exposición laboral al látex.

rio general; además hay que considerar que la sensibilidad de la prueba cutánea utilizada es superior a la detección de IgE específicas, metodología utilizada en forma aislada en otros estudios.

Por otra parte, y a pesar que la atopia se ha invocado como factor de riesgo para sensibilización a látex, no tuvimos diferencias significativas en cuanto a antecedente de enfermedades atópicas en ambos grupos, sensibilizados y no sensibilizados al látex, llamando eso sí la atención el alto porcentaje de individuos que refirieron antecedentes atópicos en los dos grupos.

El hecho de no haber encontrado más síntomas de alergia en el grupo sensibilizado durante la exposición laboral, en forma significativa en comparación con el grupo no sensibilizado, podría ser explicado por el número reducido de individuos estudiados. Es necesario, a futuro, aclarar si esta tendencia se mantiene aumentando la cantidad de pacientes estudiados. También es posible plantear que existan otras sustancias, de contacto directo o en suspensión, que puedan actuar como alergenos o como irritantes, en este ambiente.

Es posible plantear que el fenómeno observado respecto a la disminución del porcentaje de sensibilización en el grupo de más de 31 a 40 h de exposición

\section{REFERENCIAS}

1. Stern G. Uberempfindlichkeit gegen kaustchuk als urasche von Urticaria und quinckeschem odem. Klin Wochenschrift 1927; 6: 1096-7.

2. Nutter AF. Contact urticaria to rubber. Br J Dermatol 1979; 101: 597-8.

3. Subramaniam A. The chemistry of natural rubber latex. In: Latex Allergy. ED: Fink NJ. Immunol Allergy Clin North Am 1995; 15: 1-21. semanal al látex corresponda al desarrollo de un mecanismo de tolerancia, ya que este grupo no presentaba un promedio de edad mayor a los otros grupos, lo que hubiera podido explicar una disminución de su reactividad cutánea. También podría invocarse como posible factor causal de este hecho, que en este grupo pudieran haber existido traslados a otros servicios debido a síntomas en relación a la exposición al látex, lo que no había ocurrido. Ya que no había diferencias significativas entre los grupos en cuanto a edad, no podemos invocar las jubilaciones como factor causal.

Sin duda, estos resultados plantean importantes desafíos en relación con las medidas preventivas que deben tomarse en el personal de salud en general. El personal de salud ya sensibilizado al látex, pero sin evidencia clínica de alergia, debe en lo posible disminuir el grado de exposición a elementos de látex tanto en su medio laboral como familiar, y conocer respecto a las eventuales manifestaciones clínicas de alergia al látex. Respecto a aquellas personas sintomáticas, debe establecerse la relación causal de estos síntomas con la exposición al látex, y si ello se comprueba, facilitar un cambio en su medio laboral e intentar eventualmente inmunoterapia ${ }^{34}$ con extractos de látex estandarizados.

4. Nettis E, Colanardi MC, Ferrannini A, Tursi A. Sensitization to rubber additives. Allergy 2001; 56: 1018-20.

5. Blanco C, Almeida l, García A. Manifestaciones clínicas de la alergia a látex. En: Alergia al látex. Blanco C, Quirce S. MRA Ed. Barcelona 2002; 45-62.

6. Quirce S, Fernández-Nieto M, De Miguel J, Sastre J. Chronic cough due to latex-induced eosinophilic bronchitis. J Allergy Clin Immunol 2001; 108: 143.

7. Lagier F, Vervloet D, Lhermet I, Poyen D, Charpin D. Prevalence of latex allergy in operating room 
nurses. J Allergy Clin Immunol 1992; 90: 319-22.

8. Carrillo T, Blanco C, Quiralte J, Castillo R, Cuevas M, Rodríguez De Castro F. Prevalence of latex allergy among greenhouse workers. J Allergy Clin Immunol 1995; 96: 699-701.

9. Conde-Salazar L, Miranda Romero A. Caucho y gomas: Dermatosis por gomas y sus derivados. En: Dermatitis de Contacto. JM Camarasa et al. Madrid. Ed. Aula Médica 1999; 225-36.

10. Conde-Salazar L, Del Río E, Guimaraens D, GonzáLEZ D. Type IV allergy to rubber additives. A 10 years study of 686 cases. J Am Acad Dermatol 1993; 29: 176-80.

11. Moneret-Vautrin Da, Beaudouin E, Widmer S, Mouton C, Kanny G, Prestat F et al. Prospective study of risk factors in natural rubber latex hypersensitivity. J Allergy Clin Immunol 1993; 92: 668-77.

12. Nel A, Gujuluva C. Latex antigens: identification and use in clinical and experimental studies, including cross reactivity with food and pollen allergens. Ann Allergy Asthma Immunol 1998; 81: 388-98.

13. Brehler R, Theissen U, Mohr C, Luger T. Latex-fruit syndrome: frequency of cross-reacting IgE antibodies. Allergy 1997; 52: 404-10.

14. Domínguez J, Martínez-Cocera C, Bartolomé JM. Alergia al látex. En: Guía de alergia para residentes y atención primaria. Ed. Díaz de Santos. Pelta R., Gandolfo M. Madrid 2001; 185-94.

15. Hunt LW, Fransway AS, Reed CE, Miller LK, Jones RT, SWAnson MC et al. An epidemic of occupational allergy to latex involving health care workers. J Occup Environ Med 1995; 37: 1204-9.

16. Luss G, Sussman G, Deal K, Brown S, Cinidino M, Siu S et AL. Latex allergy: epidemiological study of 1,351 hospital workers. Occup Environ Med 1997; 54: 335-42.

17. Sussman GL, Liss GM, Deal K, Brown S, Cinidino M, Siu $S$ ET AL. Incidence of latex sensitization among latex glove users. J Allergy Clin Immunol 1998; 101: 171-8.

18. Tarlo S, Sussman GL, Holness DL. Latex sensitivity in dental students and staff: A cross-sectional study. J Allergy Clin Immunol 1997; 99: 396-401.

19. Kelly KJ, Pearson ml, Kurup VP, Havens Pl, Byrd RS, Setlock MA et al. A cluster of anaphylactic reactions in children with spina bifida during general anesthesia: epidemiologic features, risk factors and latex hypersensitivity. J Allergy Clin Immunol 1994; 94: 53-61.

20. Ownby DR, Tomlanovich, Sammons N, McCullough J. Anaphylaxis associated with latex allergy during barium enema examinations. Am J Roentge- nol 1991; 156: 903-8.

21. Slater JE. Rubber anaphylaxis. N Eng J Med 1989; 27: 1126-30.

22. Thompson RL. Educational challenges of latex protein allergy. En: Fink J editor. Latex allergy. Philadelphia: Saunder. Immunol Allergy Clin North Am 1995; 15: 159-74.

23. Hetier JM. Natural latex 1991; economic aspects. Clin Rev Allergy 1993; 11: 299-307.

24. Tomazic V, Shampaine E, Lamanna M, Withrow T, Adkinson F, Hamilton R. Cornstarch powder on latex products is an allergen carrier. J Allergy Clin Immunol 1994; 93: 751-8.

25. Lundberg M, Wrangsjo K, Johansson SGO. Latex allergy from glove powder-an unintended risk with the switching from talc to cornstarch. Allergy 1997; 52: 1222-8.

26. Vicherat L, Arredondo SM, Zagolin M, Arredondo S, Pizarro F. Hipersensibilidad al látex en personal de la salud. Rev Chilena de Enfermedades Respiratorias 1997; 13: 278.

27. Turjanmaa K, Alenius H, Makinen-Kimjunen S, ReunaLA T, Palosuo T. Natural rubber allergy. Allergy 1996; 51: 593-602.

28. Turjanmaa K, Reunala T, Rasanen L. Comparison of diagnostics methods in latex surgical glove contact urticaria. Contact Dermatitis 1988; 19: 241-7.

29. HamitTon RG, ADKINSON NF JR. Diagnosis of natural rubber latex allergy: multicenter latex skin testing efficacy study. Multicenter latex skin testing study task force. J Allergy Clin Immunol 1998; 102: 482-90.

30. Blanco C, Carrillo t, Ortega N, Alvarez M, DomínGUEZ C, CASTILLO R. Comparison of skin prick test and specific serum IgE determination for the diagnosis of latex allergy. Clin Exp Allergy 1998; 28: 971-6.

31. Charous Bl, Hamilton RG, Yunginger JW. Occupational latex exposure: characteristic of contact and systemic reactions in 47 workers. J Allergy Clin Immunol 1994; 94: 12-8.

32. Jaeger D, Kleinhans D, Czuppon AB, Baur X. Latexspecific proteins causing immediate-type cutaneous, nasal, bronchial and systemic reactions. J Allergy Clin Immunol 1992; 89: 759-68.

33. Garabrant D, Schweitzer S. Epidemiology of latex sensitization and allergies in health care workers. J Allergy Clin Immunol 2002; 110: S82-S95.

34. Tabar A, Gómez B, Arroabarren E, Rodríguez M, Lázaro L, Anda M. Perspectivas de tratamiento de la alergia al látex: inmunoterapia. An Sist Sanit Navar 2003; 26 Suppl 2: 97-102. 